\title{
Corela
}

Cognition, représentation, langage

HS-16 | 2015

Diversité des pratiques de recherche en science du langage

\section{Un exemple du probleme de la description}

Le modèle de la déclinaison gréco-latine dans la grammaire de Jérôme Megiser, Institutionum linguae turcicae (1612)

\section{Claire Özkal}

\section{(2) OpenEdition}

\section{Journals}

Édition électronique

URL : http://journals.openedition.org/corela/3771

DOI : $10.4000 /$ corela.3771

ISSN : 1638-573X

Éditeur

Cercle linguistique du Centre et de l'Ouest - CerLICO

Référence électronique

Claire Özkal, « Un exemple du probleme de la description », Corela [En ligne], HS-16 | 2015, mis en ligne le 10 juillet 2015, consulté le 01 mai 2019. URL : http://journals.openedition.org/corela/3771 ; DOI 10.4000/corela.3771

Ce document a été généré automatiquement le 1 mai 2019.

\section{c) (i)(2)}

Corela - cognition, représentation, langage est mis à disposition selon les termes de la licence Creative Commons Attribution - Pas d'Utilisation Commerciale - Partage dans les Mêmes Conditions 4.0 International. 


\section{Un exemple du probleme de la description}

Le modèle de la déclinaison gréco-latine dans la grammaire de Jérôme Megiser, Institutionum linguae turcicae (1612)

\section{Claire Özkal}

\section{Quelques particularités du turc}

1 Avant d'aborder la question du nom dans les grammaires du $17^{\mathrm{e}}$ siècle, nous souhaiterions exposer les aspects de la langue turque qui nous intéressent dans le cadre de cette description, et plus particulièrement sa déclinaison. Jean Deny, dans son imposante grammaire (1921), décrit ainsi les cas du nom :

Si l'on fait abstraction du cas absolu, on peut considérer que la déclinaison de l'osmanli' ${ }^{1}$ comporte cinq cas: le génitif (en turc اظافت izafet " annexion »), l'accusatif ( مفعول به mef ülün bih « qui supporte l'action »), le datif ( مفعول اليه mef ülün ileyh “ auquel l'action est destinée »), l'ablatif (مفعول عنه mef' ūlün anh « duquel émane l'action»), le locatif (مفعول فيه (مفيه mef' ülün fih «dans lequel l'action a lieu »). On peut y ajouter le cas relatif dont il sera question plus loin. (Deny, $1921: 170$ )

2 La description de Deny, qui marque la stabilisation d'une conception de la flexion casuelle avec des cas communs à ceux du latin (nominatif, accusatif, génitif, datif, ablatif), exclut le vocatif et mentionne le locatif. Nous n'aborderons pas le cas relatif car, comme pour d'autres cas cités par certaines grammaires, tels le sociatif, l'équatif, le munitif et le privatif, il y a débat tant chez les grammairiens turcs que chez les grammairiens étrangers sur le statut de ces suffixes, considérés la plupart du temps comme dérivationnels et parfois casuels. Nous nous bornerons donc aux suffixes casuels « admis de tous ${ }^{2} »$ : nominatif, accusatif, génitif, datif, ablatif et locatif. Deny distingue alors deux déclinaisons : 


\begin{tabular}{|c|c|c|c|c|}
\hline & \multicolumn{2}{|c|}{$\begin{array}{l}\text { Noms dont la dernière voyelle est } \\
\text { antérieure }(e, i, \ddot{o}, \ddot{u})\end{array}$} & \multicolumn{2}{|c|}{$\begin{array}{l}\text { Noms dont la dernière voyelle est } \\
\text { postérieure }(a, 1, o, u)\end{array}$} \\
\hline $\begin{array}{l}\text { Cas absolu ou } \\
\text { nominatif }\end{array}$ & \multirow{6}{*}{$\begin{array}{l}\varnothing \varnothing \\
-i n-i n \\
-i-i \\
-e-e \\
-d e n-d e n \\
-d e-d e\end{array}$} & \multirow{6}{*}{$\begin{array}{l}\varnothing \varnothing \\
-\ddot{u} n-\ddot{n} \\
-\ddot{u}-\ddot{u} \\
-e-e \\
-d e n-d e n \\
-d e-d e\end{array}$} & \multirow{6}{*}{$\begin{array}{l}\varnothing \varnothing \\
-1 n-1 n \\
-1-1 \\
-a-a \\
-d a n-d a n \\
-d a-d a\end{array}$} & \multirow{6}{*}{$\begin{array}{l}\varnothing \varnothing \\
-u n-u n \\
-u-u \\
-a-a \\
-d a n-d a n \\
-d a-d a\end{array}$} \\
\hline Génitif & & & & \\
\hline Accusatif & & & & \\
\hline Datif & & & & \\
\hline Ablatif & & & & \\
\hline Locatif & & & & \\
\hline Pluriel & \multicolumn{2}{|c|}{ Nom + -ler + suffixe casuel } & \multicolumn{2}{|c|}{ Nom + -lar + suffixe casuel } \\
\hline
\end{tabular}

Tableau 1 : Déclinaison du nom d'après Deny (1921)

3 Ce tableau fait apparaître l'alternance vocalique des désinences casuelles, qui est motivée par la loi d'harmonie vocalique :

Les voyelles du mot turc sont soumises à l'action de l'harmonie vocalique qui n'est qu'un cas particulier d'un phénomène phonétique plus général, l'assimilation. Du jeu de ce phénomène se dégage notamment une loi très importante qui peut être énoncée ainsi : un seul et même mot turc contient soit uniquement des voyelles de la série antérieure: e, i, ö, ü ; soit uniquement des voyelles de la série postérieure : a, 1, o, u. (Deny, $1921: 105$ )

4 Il s'agit d'un phénomène que l'on ne retrouve pas dans les langues romanes. Par conséquent, sa description semble difficile dans le cadre de la tradition grammaticale gréco-latine. Autant le modèle de la déclinaison gréco-latine semble favoriser la description du nom en turc puisque l'histoire montre que la description de la déclinaison s'est stabilisée, contrairement au nom en français, autant elle ne semble pas permettre une description complète de la morphologie des mots turcs déclinables.

\section{Premières descriptions du turc : Argenti (1533) et Postel (1575)}

\subsection{La regola del parlare turcho, Filippo Argenti (1533)}

5 Le premier exemple de déclinaison du nom est celui proposé par la grammaire manuscrite italienne d'Argenti (1533) ${ }^{3}$.

\begin{tabular}{|l|l|l|}
\hline & Singulier & Pluriel \\
\hline
\end{tabular}




\begin{tabular}{|l|l|l|l|l|}
\hline Nominatif & poeta & nami $^{4}$ & li poeti & namilar \\
Génitif & del poeta & naminum & dei poeti & namilarun \\
Datif & al poeta & namia & alli poeti & namilara \\
Accusatif & poeta & namii & - & - \\
Vocatif & o poeta & iuh nami & o poeti & iuh namilar \\
Ablatif & dal poeta & namidan & da poeti & lamilardan \\
\hline
\end{tabular}

Tableau 2 : Déclinaison du nom d'après Argenti $(1533)^{5}$

6 Dans cet exemple, qui ne comporte ni définition, ni explication, l'italien (langue de référence) est utilisé comme modèle pour décliner le nom; dès lors, c'est le modèle de la tradition grammaticale gréco-latine qui est appliqué. Cela explique l'absence de locatif et la présence d'une forme pour le vocatif. L'auteur ne précise rien sur cette forme, composée, comme l'italien, d'une interjection.

\subsection{Instruction des motz de la langue turquesque, Guillaume Postel} (1575)

\begin{tabular}{|l|l|l|l|l|}
\hline & \multicolumn{2}{|l|}{ Singulier } & \multicolumn{2}{l|}{ Pluriel } \\
\hline Nom. & l'homme & er & les hommes, homines & erler ou adamlar \\
Gén. & de l'homme, hominis & erun & des hommes & erlerun ou adamlerun \\
Dat. & à l'homme & era & aux hommes & erlera ou adamlara \\
Acc. & l'homme, hominem & eri & les hommes & erleri ou adamlari \\
Abl. & de l'homme & erdan & des hommes, ab hominibus & erlerdan ou adamlerdan \\
\hline
\end{tabular}

Tableau 3 : Déclinaison du nom d'après Postel (1575)

7 La déclinaison que propose Postel ne fait état ni d'un vocatif ni d'un locatif. Cette absence peut s'expliquer par le fait que sa langue de référence est d'abord le français. Or, il fait néanmoins appel au latin pour distinguer deux formes turques pour lesquelles la traduction française n'est pas satisfaisante car équivalente. Ainsi au singulier, pour distinguer le nominatif de l'accusatif, il cite la traduction latine à l'accusatif de son exemple et pour distinguer le génitif de l'ablatif, il procède de la même manière en traduisant le génitif latin. Il utilise le même procédé pour le pluriel en inversant ces exemples : il donne la traduction en latin du nominatif pour le distinguer de l'accusatif et la traduction latine de l'ablatif pour le distinguer du génitif. Postel ne cite pas les cas et sa description est faite sous forme linéaire au sein du texte. Le latin vient donc ici au secours d'un dispositif descriptif inadéquat que constitue le paradigme de la déclinaison du nom en français.

8 Pour le pluriel, Postel précise que « la pluralité des Noms \& des Pronoms, comme la tierce personne du verbe se finit en ler ou lar » (Postel, $1575: \mathrm{np}$ ). Il distingue donc deux formes du pluriel, indique qu'il s'agit de la même pour la conjugaison des verbes mais n'explique pas les conditions qui motivent l'apparition de l'une ou l'autre forme. Les exemples de pluriel donnés par Postel illustrent la confusion autour de ce phénomène puisque le pluriel du nom «adam» est noté soit -ler soit -lar. Pour sa part, Argenti ne donne pour exemple qu'un seul pluriel pour le nom, celui du suffixe -lar. 
Ces premiers textes, qui ne sont pas véritablement considérés comme des grammaires, illustrent comment se construit la description du nom d'après le modèle de la tradition grammaticale gréco-latine : la flexion casuelle comporte les six cas du latin et l'alternance vocalique est soit passée sous silence, soit évoquée succinctement. Postel se distingue par l'absence du vocatif et l'annonce des deux formes du pluriel mais son texte, d'une vingtaine de pages, n'apporte pas plus d'éléments.

\section{Megiser (1612) : tradition et modernité d'une description}

Megiser, philologue qui a publié plusieurs dictionnaires multilingues, fait paraître en 1612 la première grammaire du turc imprimée en Europe ${ }^{6}$. Elle s'inscrit dans la tradition : écrite en latin, elle porte un titre classique ${ }^{7}$ et est divisée en parties du discours. Megiser traite d'abord, des parties déclinables (nom, pronom, verbe, participe) puis des parties indéclinables (adverbe, préposition, conjonction, interjection). Le nom est décrit à partir des «accidents du nom» de la tradition grammaticale gréco-latine: le genre (le grammairien précise que cela n'existe pas en turc), le cas, le nombre, l'espèce et la figure. La déclinaison occupe une place importante dans le chapitre sur le nom, l'auteur y consacre plusieurs pages.

\subsection{Une déclinaison « classique »}

\begin{tabular}{|l|l|l|l|l|}
\hline \multicolumn{3}{|l|}{ Singulier } & \multicolumn{2}{l|}{ Pluriel } \\
\hline Nom. & Homo & Adem & Homines & Ademlar \\
Gén. & Hominis & Ademung & Hominum & Ademlarung \\
Dat. & Homini & Ademe & Hominibus & Ademlare \\
Acc. & Hominem & Ademi & Homines & Ademlari \\
Voc. & ô Homo & ja Adem & ô Homines & ja Ademlar \\
Abl. & ab Homine & Ademdan & Hominibus & Ademlardan \\
\hline
\end{tabular}

Tableau 4 : Déclinaison du nom d'après Megiser (1612)

\subsubsection{Remarque préliminaire : la transcription}

11 En comparant le paradigme proposé par Megiser avec celui de Postel, nous remarquons d'emblée des différences sur l'orthographe du nom décliné, qui révèlent certaines difficultés auxquelles ont été confrontés les deux auteurs. En effet, ce mot si commun qu'est « homme » ou « adam » en turc est transcrit de deux façons. Megiser écrit « adem » lors de la déclinaison mais aussi «adam» comme Postel, dans d'autres passages de sa grammaire. On remarque aussi une différence au génitif avec la désinence -un chez Postel et -ung chez Megiser. Ces disparités sont liées au problème posé par la transcription: d'une part, parce qu'elle est réalisée à partir de l'oral, avec les problèmes d'accent qu'elle suppose $^{8}$; d'autre part, parce qu'il n'existe pas de norme de transcription à partir de l'alphabet arabe, utilisé pour le turc ${ }^{9}$. Ainsi, si Megiser ne donne pas une transcription unique du mot "adam », on peut supposer qu'il s'agit soit d'une mauvaise interprétation des sources orales, soit d'une transcription erronée du mot en caractères arabes (qui dans 
sa graphie ne permet pas la distinction entre «adam » et «adem », la deuxième voyelle n'étant pas écrite). Pour la désinence du génitif, nous pouvons considérer qu'il s'agit du même problème. Megiser semble plus rigoureux pour le pluriel: on retrouve systématiquement la désinence -lar dans cet exemple.

\subsubsection{Un vocatif « fabriqué »}

12 Megiser expose les six cas latins : le locatif est donc absent du paradigme et l'on trouve une forme pour le vocatif. La forme proposée par l'auteur interpelle : elle est différente de celle proposée par Argenti et se distingue des autres cas en étant composée du nom au nominatif et de ce que Megiser nomme « un petit mot». L'auteur explique ainsi cette forme :

Le vocatif conserve la terminaison du nominatif. Le petit mot Ia, qui est turc, est placé devant, comme Ia Beg, bien que ceux qui parlent avec distinction, utilisent souvent un vocatif persan comme $\mathrm{j}$ et de nombreux autres. ${ }^{10}$

13 Cette construction d'une forme vocative que Megiser introduit dans la déclinaison est révélatrice de ce que peut constituer un cadre grammatical : c'est un modèle que l'on suit et pour lequel, dans cet exemple, ce n'est pas le modèle qui s'adapte à la langue mais bien le contraire. Megiser crée donc une forme qui s'adapte au modèle de la tradition grammaticale gréco-latine et qui survivra tant que ce modèle n'aura pas été remis en question ${ }^{11}$.

\subsubsection{La désinence du locatif comme préposition}

14 La désinence du locatif -da/-de est traitée au sein du chapitre consacré aux prépositions ${ }^{12}$. Megiser introduit son chapitre en justifiant l'existence de cette partie du discours, tout en remarquant que les prépositions sont postposées au nom:

Chez les Turcs, elle [la préposition] est utilisée : ainsi pratiquement toutes les prépositions sont placées après les noms, et avec presque nul autre cas que ceux qui construisent le nom. ${ }^{13}$

15 Ce chapitre est constitué de la liste des prépositions latines pour lesquelles le grammairien propose l'équivalent en turc. En début de chapitre, comme traduction de la préposition latine " ad ", l'auteur donne le suffixe du datif-a/-e qui a été exposé plus tôt dans la déclinaison du nom. Comme traduction de la préposition «apud», il propose ce qu'il nomme «les prépositions »-da et -de. Il ajoute un peu plus loin qu'elles sont aussi l'équivalent de la préposition latine « in ». Les désinences casuelles du locatif sont donc traitées comme des prépositions.

D'autres grammairiens, confrontés au même problème pour d'autres langues, ont eu recours au même procédé. Kozma Ahacič (2008) décrit un phénomène analogue dans la première grammaire du slovène, Arcticae horulae succisivae de Latinocarniolana literatura (1584) d'Adam Bohoric :

[...] his description [la description de Bohorič (BH 1584, p. 44) omits the two cases nowadays called the locative and instrumental, since they "are not in the grammar" - that is, in the Latin (or Greek, or Hebrew) grammar accessible to him. The Latin ablative, however, is preserved since Slovenian "has it in the grammar" - that is, since Slovenian is capable of expressing it 
with the preposition "od" ("from") and the genitive. This, however, is not so say that the locative and instrumental were overlooked. Bohoric proposes a solution for the two cases in his chapter on the preposition. ${ }^{14}$ prépositions latines, en vient donc à ranger dans cette catégorie tout élément équivalent sémantiquement. Les traductions des prépositions latines « $\mathrm{a}$ », « $\mathrm{ab}$ », « de », « ex» correspondent alors aux désinences de l'ablatif-dan/-den. Il n'y a que pour ce dernier cas que le grammairien indique qu'il s'agit de l'ablatif du nom : «cette locution est surtout l'ablatif du nom ${ }^{15}$ ». Dans son chapitre sur le nom, il indiquait déjà pour l'ablatif qu'il « se forme par l'ajout de la préposition den, laquelle signifie $a b$ ou $a$, Begden, a Domino ${ }^{16}$ ». Cependant, il ne fait pas la même observation pour les marques du datif.

En choisissant de traiter ce chapitre à partir des prépositions latines, il crée un nouveau problème. Les désinences du datif et de l'ablatif, autrement dit les marques morphologiques de la flexion casuelle $\mathrm{du}$ nom, sont assimilées à la catégorie grammaticale des prépositions, comme il l'énonce pour l'ablatif. Comment justifier que ces «particules ", terme de l'auteur, apparaissent dans deux parties du discours : l'une déclinable (le nom) et l'autre indéclinable (la préposition)? Décrire le locatif comme une préposition apparaît comme une solution peu satisfaisante car en se servant du latin comme langue de référence, l'auteur ajoute une ambiguïté sur le statut des suffixes du datif et de l'ablatif. Cette confusion perdurera et sera vivement critiquée par le grammairien Redhouse (1846) qui choisit donc de traiter des désinences casuelles au sein de la partie «prépositions» :

Comme tous mes devanciers, sans aucune exception, ont cru devoir faire accorder les règles étymologiques du nom ottoman avec celles du nom latin, et ont, par conséquent, imaginé six cas dans chaque nombre, j'ai pensé qu'il serait utile de donner ici des exemples de la forme de ces prétendus cas. Ces messieurs m'ont épargné la nécessité de prouver que dans cette circonstance, ainsi que dans bien d'autres, c'est l'esprit de système qui les a égarés ; car tous ils ont ensuite rangé les terminaisons de leurs cas parmi les prépositions (ou postpositions), dans leurs chapitres sur cette partie du discours. ${ }^{17}$

Redhouse relève l'ambiguïté en fustigeant ses prédécesseurs pour avoir reproduit trop fidèlement le modèle de la déclinaison latine.

Viguier (1790) se distingue des grammairiens cités par Redhouse. Il est le premier à introduire le locatif (ou « commoratif ») en s'affranchissant du modèle :

L'affinité constante de la langue Latine \& de la Turque dans la construction, par l'inversion des termes, n'observe plus la même réciprocité dans les Déclinaisons \& les Conjugaisons. ${ }^{18}$

21 Il bouleverse le paradigme de la déclinaison et est à l'origine d'une innovation qui aura beaucoup de succès à partir de la seconde moitié du $19^{\mathrm{e}}$ siècle. Lors de son exposé sur les déclinaisons, il annonce ne pas omettre «le Commoratif ou Habitatif inusité dans les Déclinaisons latines ${ }^{19} »$. 


\subsection{Préhistoire de la loi d'harmonie vocalique}

22 S'il est intéressant de comprendre comment un grammairien adapte son modèle à son objet d'étude, il est encore plus intéressant de voir naître dans un cadre qui ne le prévoit pas, le traitement d'un phénomène étranger à la langue de référence de ce cadre. L'harmonie vocalique n'est pas un phénomène présent dans les langues romanes. Mais c'est un fait que l'on observe en hongrois, une langue connue de Megiser. Or, il semble que cela soit davantage l'observation des alternances vocaliques des suffixes casuels qui permette au grammairien de poser les jalons de la loi d'harmonie vocalique.

Megiser a lu Bartholomaeus Georgievic, qui profite de sa captivité chez les Ottomans au $16^{\mathrm{e}}$ siècle, pour écrire un court traité sur les rites et cérémonies des $\operatorname{Turcs}^{20}$. Il y fait une très brève description de la langue turque qui consiste en une liste de mots, un dialogue et une seule et unique règle qui concerne le pluriel. Cette règle indique qu'il existe deux formes pour le pluriel, -ler et -lar, qu'elles sont employées pour le nom et pour le verbe et que ce qui conditionne l'apparition de l'une ou l'autre forme est la voyelle en début du mot ou la voyelle contenue dans la première syllabe du mot :

Tu n'ignores peut-être pas, ami Lecteur, que dans tous les noms et verbes de la langue des Perses $^{21}$, où tu aurais constaté en lisant les terminaisons lar ou ler, on peut reconnaitre le nombre pluriel de la troisième personne, formé par l'ajout à la troisième personne du singulier des terminaisons lar ou ler. $\mathrm{Tu}$ observeras cette règle si tu veux former à partir du singulier le pluriel ou à partir du pluriel le singulier ${ }^{22}$.

Georgievic donne ensuite plusieurs exemples qui viennent illustrer la règle énoncée :

Si le nom commence par la voyelle a comme Adam, c'est-à-dire l'homme, ou par une syllabe en a, comme Chalam, la plume, alors on ajoute lar et ce sera le pluriel. Exemple de lar, comme Adam, l'homme, en ajoutant lar, on aura le pluriel, comme adamlar, les hommes, chalam, la plume, chalamlar, les plumes et non chalamler, iazar, il écrit, iazarlar, ils écrivent, et de la même manière pour les autres. Si toutefois le nom ou le verbe commence soit par la voyelle e ou i soit par la syllabe [en e ou i], alors [on ajoute] ler. Exemple de ler, comme echmeh, le pain, si on ajoute ler, on aura le pluriel, hechmechler, les pains : eder, il fait, ederler, ils font, tu observeras cette règle. A mon avis, cette langue des Perses est la plus facile de toutes les langues du monde ${ }^{23}$.

Postel (1575) remarque ces deux formes mais ne formule aucune règle. Megiser, qui reprend les mêmes exemples que Georgievic, va reformuler la règle et offrir les premiers éléments $d u$ principe d'harmonie vocalique. Il distingue d'emblée les voyelles «arrondies» (« rotundum ») des voyelles «fines ${ }^{24}$ »(« subtilis»). Le terme « rotundum» apparaît une première fois dans le texte, dans le chapitre consacré aux lettres, pour définir certaines consonnes :

Huit de ces lettres sont nommées «arrondies» par les Turcs parce que lors de leur prononciation elles remplissent à peu près la bouche : comme on peut le voir ح ha, ص sad, ض tzad, ط tuoi, ظ suoi, ع ain, غ gain, et ق Kaff. ${ }^{25}$

Cette remarque nous permet de comprendre que cet adjectif renvoie à la prononciation de la lettre. Il n'explicite ce terme qu'au sein de ce chapitre. Son opposé, « subtilis », n'est pas expliqué. Le grammairien indique ainsi au début de son chapitre sur les noms : 
Le nombre et les cas sont semblables à ceux des Latins. Mais ils [les noms] se terminent au nominatif des noms, soit par des voyelles, lesquelles sont fines ou arrondies, soit par des consonnes. ${ }^{26}$

Autrement dit, il introduit d'emblée un classement à partir d'un critère phonétique qui va permettre, dans un premier temps, la conception d'une règle pour la formation du pluriel :

La formation du nominatif pluriel est caractérisée par la syllabe Lar ou Ler : pour Lar du moins, si le singulier au nominatif est l'une des deux voyelles arrondies, a ou o : mais si celui-là comporte e, on ajoute à ce moment-là Ler : comme, Adam, homo, Adamlar, homines : Beg, dominus, Begler, Domini. ${ }^{27}$

Megiser ajoute plus loin que la règle est la même pour la formation du pluriel des verbes. A l'occasion de son exposé sur les cas du nom, il généralise cette règle pour les deux formes du datif $(-a /-e)$ et de l'ablatif (-den/-dan) ainsi que pour la marque du locatif, traitée comme une préposition. Même si, dans cet exemple, le grammairien ne se montre pas très rigoureux dans l'application de cette règle et va même jusqu'à indiquer la possibilité d'utiliser les deux formes de l'ablatif et du pluriel pour certains noms, il est indéniable que Megiser met le doigt sur un phénomène qui est absent des descriptions que l'on trouve chez ses successeurs du $17^{\mathrm{e}}$ siècle.

Il semble que Megiser se soit largement inspiré de la règle de son prédécesseur Georgievic, qu'il cite régulièrement à propos du vocabulaire turc, pour en proposer une plus pertinente. Il est intéressant de noter que ces observations sont totalement absentes des quelques ouvrages postérieurs (Du Ryer (1633), Molino (1640), Pétis de la Croix (1660), Seaman (1670)), qui, sur le sujet, sont soit très brefs (Molino), soit muets car la transcription n'est pas systématique (Du Ryer, Pétis de la Croix, Seaman). En effet, ce qui rend le phénomène "visible » est l'usage d'une transcription en alphabet latin qui fait apparaitre les voyelles de suffixes, invisibles dans leur écriture d'origine, en alphabet arabe. Ceci peut expliquer l'absence de remarque sur l'alternance vocalique pour les grammairiens qui conservent l'alphabet d'origine dans les exemples. On a certainement ici la préhistoire de la notion d'harmonie vocalique ${ }^{28}$.

\section{D'autres horizons : cas de l'arabe et du finnois}

30 Ce que nous observons avec le turc peut être considéré comme un phénomène généralisé dans les grammaires du $17^{\mathrm{e}}$ siècle. La Grammaire latine étendue impose un modèle et force les grammairiens à adapter leur objet à ce modèle.

Nous avons choisi de reporter ici ce que l'on trouve dans une grammaire du finnois, Linguae Finnicae brevis institutio d'Eskil Petraeus (1649) et une grammaire de l'arabe, nstitutiones linguae arabicae de Petro Metoscita (1624), autour des deux questions qui nous intéressent dans le cadre de la description du nom : le traitement des cas et le vocatif. Les exemples du traitement du nom du finnois et de l'arabe nous ont paru particulièrement pertinents car ils présentent, d'après les grammaires modernes, pour l'un, quinze cas et pour l'autre, trois cas.

Les exemples et commentaires sur la grammaire du finnois sont entièrement empruntés à l'article de Mikko Korhonen ${ }^{29}$. 


\begin{tabular}{|c|c|c|}
\hline & $\begin{array}{l}\text { Linguae Finnicae brevis institutio, Eskil Petraeus } \\
(1649)\end{array}$ & $\begin{array}{l}\text { Institutiones linguae } \\
\text { arabicae, Petro Metoscita } \\
(1624)\end{array}$ \\
\hline $\begin{array}{l}\text { Les cas } \\
\text { traditionnels } \\
\text { décrits dans les } \\
\text { grammaires } \\
\text { modernes }\end{array}$ & $\begin{array}{l}\text { Pour le finnois, } 15 \text { cas : nominatif, génitif, } \\
\text { partitif, accusatif, inessif, élatif, illatif, adessif, } \\
\text { ablatif, allatif, essif, translatif, instructif, } \\
\text { comitatif, abessif. }\end{array}$ & $\begin{array}{l}\text { Pour l'arabe, } 3 \text { cas : } \\
\text { nominatif, accusatif, } \\
\text { génitif. }\end{array}$ \\
\hline $\begin{array}{l}\text { Cas présentés par } \\
\text { les grammaires du } \\
17^{\mathrm{e}} \text { siècle }\end{array}$ & $\begin{array}{l}\text { Les six cas latins: nominatif, génitif, datif, } \\
\text { accusatif, vocatif, ablatif }\end{array}$ & $\begin{array}{l}\text { Les six cas latins : } \\
\text { nominatif, génitif, datif, } \\
\text { accusatif, vocatif, ablatif }\end{array}$ \\
\hline $\begin{array}{l}\text { Aménagement avec } \\
\text { le cadre de la } \\
\text { tradition } \\
\text { grammaticale } \\
\text { gréco-latine: le } \\
\text { nombre de cas }\end{array}$ & $\begin{array}{l}\text { Certains suffixes sont considérés comme des } \\
\text { adverbes (comitatif, instructif, abessif), } \\
\text { d'autres, comme le translatif, sont décrits } \\
\text { comme des particules. Pour les autres cas, } \\
\text { Petraeus les rattache aux cas latins (à } \\
\text { l'accusatif: accusatif + partitif, à l'ablatif: } \\
\text { ablatif, élatif, inessif, adessif). L'auteur de } \\
\text { l'étude sur cette grammaire en conclut que } \\
\text { pour Petraeus, le cas est une fonction } \\
\text { sémantico-syntaxique et non une structure } \\
\text { morphologique. }\end{array}$ & $\begin{array}{l}\text { Le grammairien indique } \\
\text { que les cas génitif, datif } \\
\text { et ablatif ont la même } \\
\text { désinence. }\end{array}$ \\
\hline $\begin{array}{l}\text { Aménagement avec } \\
\text { le cadre de la } \\
\text { tradition } \\
\text { grammaticale } \\
\text { gréco-latine: } \\
\text { vocatif }\end{array}$ & $\begin{array}{l}\text { Le vocatif, qui n'est pas un cas du finnois, est } \\
\text { marqué par un nom seul à la même forme que } \\
\text { le nominatif. }\end{array}$ & $\begin{array}{l}\text { Le grammairien } \\
\text { construit un vocatif à } \\
\text { partir du nom au } \\
\text { nominatif et de } \\
\text { «l'adverbe } ا \text { ي[ya]. }\end{array}$ \\
\hline
\end{tabular}

Tableau 5 : Traitement des cas d'après Petraeus (1649), Metoscita (1624)

33 Pour l'arabe comme pour le finnois, on retrouve les six cas latins. Les grammairiens aménagent ce cadre de deux façons: pour le finnois, à de nombreuses désinences correspond un seul cas, pour l'arabe, c'est l'opération inverse, plusieurs cas ont la même désinence. Dans les deux exemples, le vocatif est construit à partir de la forme du nom au nominatif. Pour l'arabe, le grammairien ajoute ce qu'il appelle « un adverbe »: procédé que l'on retrouve dans les grammaires classiques du turc et du français.

\section{En guise de conclusion}

34 L'étude du traitement de la déclinaison dans la grammaire de Megiser a montré que le modèle de la déclinaison gréco-latine imposait l'introduction du vocatif au sein des cas et empêchait la description du locatif. La perspective historique tend à confirmer que le 
locatif et le vocatif ont un destin lié. D'après les données du tableau ci-dessous, on constaste que :

1. Dans la plupart des cas, si le vocatif apparait dans le paradigme, le locatif en est absent

2. Viguier (1790) est le premier à intégrer le locatif (ou « commoratif ») et à évincer le vocatif.

3. La description ne se stabilise qu'à partir de la seconde moitié du $19^{\mathrm{e}}$ siècle.

\begin{tabular}{|c|c|c|}
\hline & $\begin{array}{l}\text { Le locatif comme } \\
\text { désinence casuelle }\end{array}$ & $\begin{array}{l}\text { Le vocatif } \\
\text { comme cas }\end{array}$ \\
\hline $17^{\mathrm{e}}$ siècle & \multirow[b]{2}{*}{$\varnothing$} & \multirow[b]{2}{*}{$\checkmark$} \\
\hline $\begin{array}{l}\text { Megiser (1612), Du Ryer (1633), Molino (1641), Pétis de la } \\
\text { Croix (1660), Maggio (1670), Seaman (1670), Meninski (1680) }\end{array}$ & & \\
\hline $18^{\mathrm{e}}$ siècle & \multirow[b]{2}{*}{$\varnothing$} & \multirow[b]{2}{*}{$\checkmark$} \\
\hline $\begin{array}{l}\text { Vaughan (1709), Holderman (1730), Pianzola (1789), Preindl } \\
\text { (1790), Comidas de Carbognano (1794) }\end{array}$ & & \\
\hline Viguier (1790) & $s$ & $\varnothing$ \\
\hline $19^{e}$ siècle & \multirow[b]{2}{*}{$\varnothing$} & \multirow[b]{2}{*}{$\checkmark$} \\
\hline $\begin{array}{l}\text { Jaubert (1833), Hindoglou (1834), Davids (1836), Boyd (1842), } \\
\text { Redhouse (1846), Timoni (1854), Dieterici (1854), Viotte } \\
\text { (1856), Dubeux (1856), Said (1877) }\end{array}$ & & \\
\hline Schroeder (1835), Barker (1854), Chodzko (1854) & $\varnothing$ & $\varnothing$ \\
\hline Pfizmaier (1847), Mallouf (1862), Youssouf (1892) & $\checkmark$ & $\varnothing$ \\
\hline Redhouse (1892) & $\checkmark$ & $\checkmark$ \\
\hline $20^{\mathrm{e}}$ siècle (avant la réforme) & \multirow{2}{*}{$\checkmark$} & \multirow{2}{*}{$\checkmark$} \\
\hline Frère Eustache Louis (1907) & & \\
\hline Dal Medico (1908), Deny (1921) & $s$ & $\varnothing$ \\
\hline
\end{tabular}

Tableau 6: Vocatif et locatif dans les grammaires du turc (1612-1921)

Malgré un cadre inadéquat, Megiser a pu décrire partiellement le principe d'harmonie vocalique. Cette découverte est à mettre en relation avec la règle du pluriel énoncée par Georgievic et l'utilisation de la transcription latine de Megiser. Si le grammairien innove et fait preuve d'une certaine finesse dans sa description, il faudra attendre la grammaire de Viguier (1790) pour que ce phénomène soit décrit comme un principe général de la langue turque.

Au-delà de l'histoire de la description du nom du turc, l'étude des problèmes posés par la Grammaire latine étendue permet de montrer que les solutions des grammairiens convergent, malgré des objets d'étude différents, pour que la langue s'adapte au modèle. 


\section{BIBLIOGRAPHIE}

ADAMOVIĆ, Milan (2009). Floransalı Filippo Argenti'nin notlarına göre (1533) 16. yüzyıl türkçesi, [Le turc du XVIe siècle d'après les notes manuscrites (1533) du Florentin Filippo Argenti], traduit du russe par Aziz Merhan, Ankara : Türk Dil Kurumu Yayınları. AHAČIČC, Kozma (2008). “ “Omnis lingua confitebitur deo” : writing the first slovenian grammar (1584) », Histoire Épistémologie Langage, 30/2, 93-112.

AKSULU, Melek (1998). Mohaç esiri Bartholomaeus Georgievic (1505-1566) ve Türklerle ilgili yazıları, [Le captif du Mohac, Bartholomaeus Georgievic (1505-1566) et ses écrits sur les Turcs], Ankara T.C. Kültür Bakanlı̆̆ı Yayınları.

AUROUX, Sylvain (1994). La Révolution technologique de la grammatisation, Liège Mardaga.

AUROUX, Sylvain (1998). « La découverte linguistique », in Pierre Fiala \& Pierre Lafon (dir.), Des mots en liberté, Mélanges Maurice Tournier, Tome I, Paris Ens éditions, 85-90.

BARKER, William Burckhardt (1854). A practical grammar of the Turkish language, Londres Bernard Quaritch.

BOYD, Charles (1842). The Turkish interpreter or a new grammar of the Turkish language, Paris Firmin Didot frères.

CHODZKO, Alexandre (1854). Le Drogman turc donnant les mots et les phrases les plus nécessaires pour la conversation, Paris Benjamin Duprat.

COMIDAS DE CARBOGNANO, Cosimo (1794). Primi principi della gramatica turca, Rome Sacrae congregationis de propaganda fide.

DAL MEDICO, Moìse (1908). Méthode théorique et pratique pour l'enseignement de la langue turque, Constantinople : V. \& H. Der-Nersessian.

DAVIDS, Arthur Lumley (1836). Grammaire Turke, traduit de l'anglais par Sarah Davids, Londres : Richard Watts.

DENY, Jean (1921). Grammaire de la langue turque (dialecte osmanli), Paris : éditions Ernest Leroux.

DIETERICI, Friedrich (1854). Chrestomathie Ottomane, Berlin : George Reimer.

DU RYER, André (1633). Rudimenta Grammatices linguae turcicae, $2^{\mathrm{e}}$ éd., Paris : Societatis Typographicæ Librorum Officij Ecclesiastici [1 $1^{\mathrm{e}}$ édition : 1630].

DUBEUX, Louis (1856). Eléments de la grammaire turque, Meulan : Imprimerie de Nicolas.

FRÈRE EUSTACHE LOUIS (1907). Grammaire complete de la langue turque comprenant les trois éléments turc, persan, arabe, Constantinople : Typographie et Lithographie Ahmed İnsan.

HINDOGLOU, Artin (1834). Grammaire théorique et pratique de la langue Turke, Paris : Imprimerie de Prosper Dondey-Dupré.

HOLDERMAN, Jean-Baptiste (1730). Grammaire turque ou méthode courte et facile pour apprendre la langue turque, Constantinople : Ibrahim Müteferrika.

JAUBERT, Pierre Amédée (1833). Eléments de la grammaire turke, $2^{\mathrm{e}}$ édition, Paris : Imprimerie Firmin Didot [ $1^{\mathrm{e}}$ édition :1823]. 
KORHONEN, Mikko (1987). «Les grammaires des langues finno-ougriennes : esquisse historique », traduit du finnois par A. Ahlquist et revu par S. Auroux, Histoire Épistémologie Langage, 9/1, 91110.

MAGGIO, Francisco Maria (1670). Syntagmaton linguarum orientalium liber secundus complectens Turcice, atque Arabica linguce Institutiones, Rome : Sacrae congregationis de propaganda fide.

MALLOUF, Nassif (1862). Grammaire élémentaire de la langue turque, Paris : Imprimerie polyglotte des éditions Blot.

MEGISER, Hieronymus (1612). Institutionum linguae turcicae libri quatuor, Leipzig.

MENINSKI, François (1680). Linguarum orientalium Turcice, Arabicœe, Persicee, Institutiones seu Grammatica Turcica, Vienne. Facsimile reprint : Istanbul : Simurg, 2000.

METOSCITA, Petro (1624). Institutiones linguae arabicae, Rome.

MOLINO, Giovanni (1641). « Brevi rudimenti del parlar turchesco », Dittionario della lingua iatliana turchesca, Rome : Apresso Antonio Maria Gioiosi.

PÉTIS DE LA CROIX, François (1660). Grammaire turque, manuscrit composé à Istanbul, Archives diplomatiques de La Courneuve, cote 83-A-13.

PFIZMAIER, Auguste (1847). Grammaire turque ou développement séparé et méthodique des trois genres de style usités, savoir l'arabe, le persan et le tartare, Vienne : Imprimerie royale.

PIANZOLA, Bernardino (1789). « Grammatiche, e colloqui per imparare le lingue italiana, grecavolgare, e turca ", Dizionario gramatiche, e dialoghi, $2^{\mathrm{e}}$ édition, Padoue : Gianantonio Conzatti, [1 $1^{\mathrm{e}}$ édition : 1781]

POSTEL, Guillaume (1575). « Instruction des motz de la langue turquesque », in Histoires orientales, Paris : Marnet et Cauellat, np.

PREINDL, Joseph de (1790). Grammaire turque d'une toute nouvelle méthode d'apprendre cette langue en peu de semaines, Berlin : s.n.

REDHOUSE, James William (1846). Grammaire raisonnée de la langue ottomane, Paris : Gide et Cie.

REDHOUSE, James William (1892). The Turkish Vade-Mecum of Ottoman colloquial langage : containing a concise ottoman grammar, $3^{\mathrm{e}}$ édition, Londres : Kegan Paul [1 ${ }^{\mathrm{e}}$ édition : 1855].

SCHROEDER, Guillaume (1835). Grammaire turque à l'usage des français et anglais, Leipzig : Othon Wigand.

SAID, Abu (1877). Turkish self-taughtor the dragoman for travellers in the east, being a new pratical and easy method of learning the Turkish language, Londres : Franz Thimm.

SEAMAN, William (1670). Grammatica linguce Turcica, Oxford : Hall Academiae Typographus.

TIMONI, Alexandre (1854). Guide de la conversation (grammaire, dialogues, vocabulaire) français- turc, Paris : Librairie orientale de Maisonneuve et Cie.

VARDAR, Berke (éd.) (2002). Açıklamalı dilbilim terimleri sözlügü [Dictionnaire commenté des termes de la linguistique], Istanbul : Multilingual yabancı dil yayınları.

VAUGHAN, Thomas (1709). A grammar of the Turkish language, Londres : J. Humfreys.

VIGUIER, Pierre François (1790). Elémens de la langue turque, ou tables analytiques de la langue turque usuelle, avec leur développement, Constantinople : Imprimerie du Palais de France.

VIOTTE, Charles (1856). Grammaire turque courte et facile, Leipzig : Théodore Steinhaussen. 
YOUSSOUF, R. (1892). Grammaire complète de la langue ottomane, Constantinople : Typographie Zareh.

\section{NOTES}

1. Les termes “ osmanli », « turc ottoman » ou « ottoman » désignent la langue turque en usage avant la réforme linguistique de 1928.

2. Le linguiste turc Berke deVardar indique, à l'article «cas» («durum») de son Dictionnaire commenté des termes de la linguistique: «Genellikle benimsenen görüse göre Türkçe'de şu durumlar vardır: yalın durum, belirtme durumu, tamlayan durumu, yönelme durumu, çıkma durumu, kalma durumu ; ayrıca bir de eşitlik durumundan söz edilir. » [Je traduis : « En général, selon l'opinion admise, on trouve ces cas dans le turc : nominatif, accusatif, génitif, datif, ablatif, locatif ; on peut citer en outre l'équatif. »] (Vardar, $2002: 84$ )

3. Le manuscrit a été reproduit en fac-similé (Adamović, 2009).

4. Les exemples turcs sont notés en italique.

5. Cf. Adamović (2009: 20).

6. Elle fut commandée par l'empereur Matthias II, roi de Hongrie, qui venait de signer un traité de paix avec les Ottomans.

7. On trouve à la même époque: Institutionum linguae graecae (Iacobi Gretseri, 1609), Institutiones linguae arabicae (Petro Metoscita, 1624), Institutiones linguae italicae (Johannes Franciscus Roemer, 1649).

8. Postel aurait appris le turc à Constantinople alors que Megiser n'aurait eu de contacts qu'avec des turcophones d'Europe.

9. Les turcs ont adopté l'alphabet arabe au moment de leur islamisation jusqu'à la réforme linguistique de 1928. Cet alphabet est aussi nommé «alphabet ottoman » en raison de la présence d'un caractère ottoman et de caractères persans.

10. «Vocativus retinet Nominativi terminationem, præposita vocula, Ia, quæ Turcica est, ut Ia Beg, quanquam ii, qui eleganter loquuntur, frequentius Vocativo Persico, $j$, utuntur, ut \& multis allis. » (Megiser, $1612: \mathrm{np})$

11. Cette remise en question est tardive. En 1836, Davids, dans sa grammaire, affirme toujours que : «Dans ses déclinaisons des noms, il [le turc] ressemble au Latin; ayant cinq cas, outre le vocatif ; mais la déclinaison osmanli est la plus régulière, parce que la radicale est retenue partout, et que les cas se trouvent formés par les terminaisons qui leur sont attachées ». (Davids, 1836 : XVLII)

12. Nous voudrions préciser que dans les descriptions modernes du turc et dans quelques grammaires anciennes, les grammairiens choisissent le terme "postposition ». Au $17^{\mathrm{e}}$ siècle, il n'est employé que dans la grammaire manuscrite de François Pétis de La Croix (1660) et dans celle de Meninski (1680).

13. « Apud Turcos usitatum est : ut omnes fere Præpositiones Nominibus postponantur, \& cum nullo propeamodum alio casu, quam Nominativo construantur. » (Megiser, 1612 : np)

14. (Ahačičc, $2008: 107$ )

15. « Atque ista locutio proprie ablativus nominis est. » (Megiser, 1612 : np) 
16. «Ablativus formatur adjectione Præpositionis den, quæ ab, vel a significat, Begden, a Domino. » (ibid.)

17. (Redhouse, $1846: 162$ )

18. (Viguier, $1790:$ IX)

19. (Viguier, $1790: X)$

20. De Turcarum ritu et caeremoniie (1544), réédité par Melek Aksulu (1998).

21. La confusion sur le nom de la langue ou de ses locuteurs est un phénomène relativement courant dans les textes du $16^{\mathrm{e}}$ siècle. Le turc est parfois désigné comme langue des Tatars.

22. «Non ignores amice lector, quod in omnibus nominibus \& verbis Persarum linguæ, ubi lar, aut ler terminationes legendo inveneris, scias plurale esse numerum tertiae personæ, formatum a tertia persona sing. numeri addita lar vel ler, terminationibus. Si vis ex singulari pluralem, vel ex plurali singulare formare, hanc observabis regula.» (Georgievic, [1544] 1998 : 135)

23. «Si nomen incipiat à vocali a ut Adam i homo, vel syllaba in a, ut Chalam i penna, tunc addes lar, \& erit pluralis. Exemplum de lar, ut Adam i homo adde lar, erit pluralis, ut adamlar i homines chalam i penna, chalamlar i penne \& non chalamler, iazar i scribit iazalar i scribunt, \& ita de aliis. Si vero ab e vel i vocalibus vel syllabis incipiat nomen aut verbum, tunc ler. Exemplum de e, ut echmech i pannis, si addas ler, erit pluralis, hechmechler i pannes : eder i facit, ederler i faciunt hanc regulam observabis. Est haec Persarum lingua omnium linguarum mundi (meo judicio) facilior. » (ibid.)

24. Ces termes n'étant pas habituels dans les grammaires de cette époque, c'est une traduction que je propose d'après le texte de Megiser.

25. "Octo hæ literæ, quod in pronunciatione os quasi implent, a Turcis rotundae appellantur : videlicet $ح$ ha, ص sad, ض tzad, b tuoi, ظ suoi, ع ain, غ gain, et ق Kaff. » (Megiser, $1612: \mathrm{np}$ )

26. « Numeri \& Casus iidem sunt, qui apud Latinos. Desinunt autem Nominativi Nominum, aut in Vocales, easque vel subtiles vel rotundas, aut in consonantes. » (ibid.)

27. «Pluralis Nominativi formatio propria est syllaba Lar, vel Ler: \& Lar quidem si alterutra vocalium rotundarum, a vel o nominativo est singulari : sin illud habet e, tum Ler adjicitur : ut, Adam, homo, Adamlar, homines : Beg, Dominus, Begler, Domini. » (ibid.)

28. Megiser n'emploie jamais ces termes, mais il décrit bien une partie de ce phénomène. C'est ce qui nous importe car "L'identité de la connaissance ne réside pas essentiellement dans la définition qu'elle peut recevoir au sein d'un système théorique donné, mais bien plutôt dans la capacité qu'a l'historien d'identifier le traitement d'une classe de phénomène et d'en suivre l'évolution. » (Auroux, 1998 : 86)

29. (Korhonen, 1987 : 91-110) 


\section{RÉSUMÉS}

Nous proposons, à travers l'étude du traitement de la déclinaison du nom dans la première grammaire européenne du turc, Institutionum linguae turcicae (1612) de Jérôme Megiser, d'exposer le double problème posé par le cadre de la tradition grammaticale gréco-latine. S'il permet de décrire les cas communs au latin et au turc, le modèle de la déclinaison latine empêche, d'une part, la présence du locatif dans le paradigme de la déclinaison (cette désinence est alors considérée comme une préposition) et impose l'introduction d'une forme vocative. D'autre part, ce modèle apparaît inadéquat pour rendre compte de l'harmonie vocalique du turc qui motive l'existence de variations pour les désinences. Or, nous verrons que Megiser se distingue pour la description de ce phénomène en utilisant des critères phonétiques, construisant ainsi la préhistoire de la notion d'harmonie vocalique.

We offer, through the study of the treatment of the declension of noun in the first European grammar of Turkish, Institutionum linguae turcicae (1612) by Jerome Megiser, to expose the dual problem posed by the Greco-Latin grammatical tradition. If it used to describe the common cases in Latin and Turkish, the pattern of the Latin declension prevents, on one hand, the presence of the locative case in the paradigm of declension (this ending is then considered as a preposition) and requires the introduction of a vocative form. On the other hand, this pattern appears inadequate for giving an account for vowel harmony in Turkish which motivates the existence of variations for endings. However, we will see that Megiser stands out with the description of this phenomenon using phonetic criteria, thus constructing the prehistory of the concept of vowel harmony.

\section{INDEX}

Mots-clés : turc, grammaire, tradition grammaticale gréco-latine, 17e siècle, flexion casuelle, harmonie vocalique

Keywords : turkish, grammar, greek-Latin grammatical tradition, 17th century, case inflection, vowel harmony

\section{AUTEUR}

CLAIRE ÖZKAL

Université Sorbonne Nouvelle - Paris 3

UMR 7597 « Histoire des Théories Linguistiques » c.ozkal@free.fr 